\title{
Virtual photon structure functions and positivity constraints
}

\author{
Ken Sasaki* \\ Department of Physics, Faculty of Engineering, Yokohama National University, Yokohama 240-8501, Japan \\ Jacques Soffer ${ }^{\dagger}$ \\ Centre de Physique Théorique, CNRS, Luminy Case 907, F-13288 Marseille Cedex 9, France \\ Tsuneo Uematsu ${ }^{*}$ \\ Department of Fundamental Sciences, FIHS, Kyoto University, Kyoto 606-8501, Japan
}

(Received 16 May 2002; published 15 August 2002)

\begin{abstract}
We study the three positivity constraints on the eight virtual photon structure functions, derived from the Cauchy-Schwarz inequality and which are, hence, model independent. The photon structure functions obtained from the simple parton model show quite different behaviors in a massive or a massless quark case, but they satisfy, in both cases, the three positivity constraints. We then discuss an inequality which holds among the unpolarized and polarized photon structure functions $F_{1}^{\gamma}, g_{1}^{\gamma}$, and $W_{T T}^{\tau}$, in the kinematic region $\Lambda^{2} \ll P^{2}$ $\ll Q^{2}$, where $-Q^{2}\left(-P^{2}\right)$ is the mass squared of the probe (target) photon, and we examine whether this inequality is satisfied by the perturbative QCD results.
\end{abstract}

DOI: 10.1103/PhysRevD.66.034014

PACS number(s): 12.38.Bx, 13.60.Hb, 13.88.+e

\section{INTRODUCTION}

The investigation of the photon structure is an active field of research both theoretically and experimentally [1]. Structure functions of unpolarized real and virtual photons $F_{2}^{\gamma}$ and $F_{\text {eff }}^{\gamma^{*}}$ have been measured through the two-photon processes in $e^{+} e^{-}$collisions as well as the resolved photon processes in the ep collider. From these data the unpolarized parton distributions in the photon were extracted in the framework of perturbative QCD (PQCD) [2]. On the other hand, there has been growing interest in the study of polarized photon structure functions $[3,4]$. Especially the first moment of the spin-dependent structure function $g_{1}^{\gamma}$ has attracted much attention in connection with its relevance to the QED and QCD axial anomaly. The next-to-leading order (NLO) QCD analysis of $g_{1}^{\gamma}$ was performed $[5,6,7]$ and, recently, the second spin-dependent structure function $g_{2}^{\gamma}$ of virtual photon has been studied in conjunction with the twist- 3 contribution [8]. For the real photon structure functions $g_{1}^{\gamma}$ and $F_{1}^{\gamma}$ there exists a positivity bound $\left|g_{1}^{\gamma}\right| \leqslant F_{1}^{\gamma}$. This bound has been analyzed recently in detail in Ref. [7].

Now we note that there appear, in total, eight structure functions in the case of a virtual photon target $[9,10,11,12]$, most of which have not been measured yet and, therefore, are unknown. In such a situation, positivity bounds would play an important role in constraining these unknown structure functions. It is well known in deep inelastic scattering off nucleon that various bounds have been obtained for the spindependent observables and parton distributions in a nucleon by means of positivity conditions [13]. In our previous paper [14] we have derived three positivity bounds, among the

\footnotetext{
*Email address: sasaki@cnb.phys.ynu.ac.jp

†Email address: soffer@cpt.univ-mrs.fr

*Email address: uematsu@phys.h.kyoto-u.ac.jp
}

eight virtual photon structure functions, which hold modelindependently. The number of positivity bounds reduces to one in the real photon case, and we have checked that this remaining bound is indeed satisfied by the structure functions obtained in the simple parton model (PM). We also presented a positivity bound for the quark distributions relevant for the spin-dependent semi-inclusive process in the two-photon reaction.

In this paper we examine the three positivity constraints on the virtual photon structure functions. By evaluating the box (a quark-loop) diagrams, we first obtain the eight virtual photon structure functions in the PM and check if they satisfy the positivity constraints or not. We then discuss an inequality which holds among the unpolarized and polarized structure functions, $F_{1}^{\gamma}, g_{1}^{\gamma}$, and $W_{T T}^{\tau}$, and we examine the PQCD results.

In the next section we discuss the eight virtual photon structure functions which were introduced by Budnev et al. [9] to describe the absorptive part of the virtual photonphoton forward scattering. The positivity constraints, which were derived in our previous paper [14] for the eight independent $s$-channel helicity amplitudes, are rewritten in terms of these structure functions. In Sec. III we calculate these eight structure functions in the PM. We find that there exists a clear difference, both in the $x$ dependence and in the magnitude, between the massive and the massless quark cases for the PM predictions. But for both cases, it turns out that the three positivity constraints are indeed satisfied for all the allowed $x$ region. In Sec. IV we study an inequality which holds among $F_{1}^{\gamma}, g_{1}^{\gamma}$, and $W_{T T}^{\tau}$ in the kinematic region $\Lambda^{2}$ $\ll P^{2} \ll Q^{2}$, where $-Q^{2}\left(-P^{2}\right)$ is the mass squared of the probe (target) photon. Since the NLO QCD results for $F_{1}^{\gamma}$ and $g_{1}^{\gamma}$ and the leading order (LO) result for $W_{T T}^{\tau}$ are already known, we will examine whether these PQCD results are consistent with this inequality. The last section is devoted to the conclusion. 


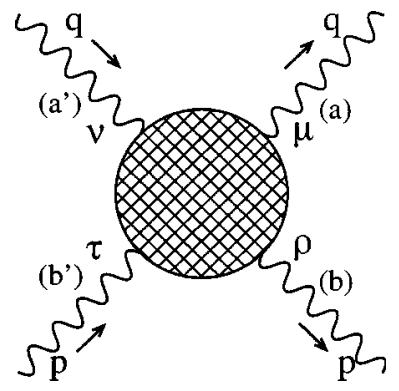

FIG. 1. Virtual photon-photon forward scattering with momenta $q(p)$ and helicities $a(b)$ and $a^{\prime}\left(b^{\prime}\right)$.

\section{PHOTON STRUCTURE FUNCTIONS AND POSITIVITY CONSTRAINTS}

We consider the virtual photon-photon forward scattering amplitude for $\gamma(q)+\gamma(p) \rightarrow \gamma(q)+\gamma(p)$ illustrated in Fig. 1:

$$
\begin{aligned}
T_{\mu \nu \rho \tau}(p, q)= & i \int d^{4} x d^{4} y d^{4} z e^{i q \cdot x} \\
& \times e^{i p \cdot(y-z)}\left\langle 0\left|T\left[J_{\mu}(x) J_{\nu}(0) J_{\rho}(y) J_{\tau}(z)\right]\right| 0\right\rangle,
\end{aligned}
$$

where $J$ is the electromagnetic current, and $q$ and $p$ are the four-momenta of the probe and target photon, respectively. The $s$-channel helicity amplitudes are related to its absorptive part as follows:

$$
W\left(a b \mid a^{\prime} b^{\prime}\right)=\epsilon_{\mu}^{*}(a) \epsilon_{\rho}^{*}(b) W^{\mu \nu \rho \tau} \epsilon_{\nu}\left(a^{\prime}\right) \epsilon_{\tau}\left(b^{\prime}\right),
$$

where

$$
W_{\mu \nu \rho \tau}(p, q)=\frac{1}{\pi} \operatorname{Im} T_{\mu \nu \rho \tau}(p, q),
$$

and $\epsilon_{\mu}(a)$ represents the photon polarization vector with helicity $a$, and $a=0, \pm 1$. Similarly for the other polarization vectors we have $a^{\prime}, b, b^{\prime}=0, \pm 1$. Due to angular momentum conservation, parity conservation, and time reversal invariance [15], we have in total eight independent $s$-channel helicity amplitudes, which we may take as

$$
\begin{gathered}
W(1,1 \mid 1,1), W(1,-1 \mid 1,-1), W(1,0 \mid 1,0), W(0,1 \mid 0,1) \\
W(0,0 \mid 0,0), \quad W(1,1 \mid-1,-1), \quad W(1,1 \mid 0,0), \\
W(1,0 \mid 0,-1) .
\end{gathered}
$$

The first five amplitudes are helicity-nonflip and the last three are helicity-flip. It is noted that the $s$-channel helicitynonflip amplitudes are semipositive, but not the helicity-flip ones.

In our previous work [14], we have applied the CauchySchwarz inequality $[16,17]$ to the above photon helicity amplitudes and have derived a positivity bound:

$$
\left|W\left(a, b \mid a^{\prime}, b^{\prime}\right)\right| \leqslant \sqrt{W(a, b \mid a, b) W\left(a^{\prime}, b^{\prime} \mid a^{\prime}, b^{\prime}\right)} .
$$

Writing down explicitly, we obtain the following three positivity constraints:

$$
\begin{array}{r}
|W(1,1 \mid-1,-1)| \leqslant W(1,1 \mid 1,1), \\
|W(1,1 \mid 0,0)| \leqslant \sqrt{W(1,1 \mid 1,1) W(0,0 \mid 0,0)}, \\
|W(1,0 \mid 0,-1)| \leqslant \sqrt{W(1,0 \mid 1,0) W(0,1 \mid 0,1)} .
\end{array}
$$

The photon-photon scattering phenomenology is often discussed in terms of the photon structure functions instead of the $s$-channel helicity amplitudes. Budnev, Chernyak, and Ginzburg (BCG) [9] introduced the following eight independent structure functions, in terms of which the absorptive part of virtual photon-photon forward scattering, $W^{\mu \nu \rho \tau}$, is written as

$$
\begin{aligned}
W^{\mu \nu \rho \tau}(p, q)= & \left(P_{T T}\right)^{\mu \nu \rho \tau} W_{T T}+\left(P_{T T}^{a}\right)^{\mu \nu \rho \tau} W_{T T}^{a} \\
& +\left(P_{T T}^{\tau}\right)^{\mu \nu \rho \tau} W_{T T}^{\tau}+\left(P_{S T}\right)^{\mu \nu \rho \tau} W_{S T} \\
& +\left(P_{T S}\right)^{\mu \nu \rho \tau} W_{T S}+\left(P_{S S}\right)^{\mu \nu \rho \tau} W_{S S} \\
& -\left(P_{T S}^{\tau}\right)^{\mu \nu \rho \tau} W_{T S}^{\tau}-\left(P_{T S}^{\tau a}\right)^{\mu \nu \rho \tau} W_{T S}^{\tau a},
\end{aligned}
$$

where $P_{i}$ 's are the following projectors:

$$
\begin{aligned}
& \left(P_{T T}\right)^{\mu \nu \rho \tau}=R^{\mu \nu} R^{\rho \tau}, \\
& \left(P_{T T}^{a}\right)^{\mu \nu \rho \tau}=R^{\mu \rho} R^{\nu \tau}-R^{\mu \tau} R^{\nu \rho}, \\
& \left(P_{T T}^{\tau}\right)^{\mu \nu \rho \tau}=\frac{1}{2}\left[R^{\mu \rho} R^{\nu \tau}+R^{\mu \tau} R^{\nu \rho}-R^{\mu \nu} R^{\rho \tau}\right], \\
& \left(P_{S T}\right)^{\mu \nu \rho \tau}=k_{1}^{\mu} k_{1}^{\nu} R^{\rho \tau}, \\
& \left(P_{T S}\right)^{\mu \nu \rho \tau}=R^{\mu \nu} k_{2}^{\rho} k_{2}^{\tau}, \\
& \left(P_{S S}\right)^{\mu \nu \rho \tau}=k_{1}^{\mu} k_{1}^{\nu} k_{2}^{\rho} k_{2}^{\tau}, \\
& \left(P_{T S}^{\tau}\right)^{\mu \nu \rho \tau}=R^{\mu \rho} k_{1}^{\nu} k_{2}^{\tau}+R^{\mu \tau} k_{1}^{\nu} k_{2}^{\rho}+k_{1}^{\mu} k_{2}^{\rho} R^{\nu \tau}+k_{1}^{\mu} k_{2}^{\tau} R^{\nu \rho}, \\
& \left(P_{T S}^{\tau a}\right)^{\mu \nu \rho \tau}=R^{\mu \rho} k_{1}^{\nu} k_{2}^{\tau}-R^{\mu \tau} k_{1}^{\nu} k_{2}^{\rho}+k_{1}^{\mu} k_{2}^{\rho} R^{\nu \tau}-k_{1}^{\mu} k_{2}^{\tau} R^{\nu \rho},
\end{aligned}
$$

with

$$
\begin{aligned}
R^{\mu \nu} & =-g^{\mu \nu}+\frac{1}{X}\left[w\left(q^{\mu} p^{\nu}+p^{\mu} q^{\nu}\right)-q^{2} p^{\mu} p^{\nu}-p^{2} q^{\mu} q^{\nu}\right] \\
k_{1}^{\mu} & =\sqrt{\frac{-q^{2}}{X}}\left(p^{\mu}-\frac{w}{q^{2}} q^{\mu}\right) \\
k_{2}^{\mu} & =\sqrt{\frac{-p^{2}}{X}}\left(q^{\mu}-\frac{w}{p^{2}} p^{\mu}\right)
\end{aligned}
$$

and $w=p \cdot q$ and $X=(p \cdot q)^{2}-p^{2} q^{2}$. Note that $R_{\mu \nu}$ is the metric tensor of the subspace which is orthogonal to $q$ and $p$, and thus $k_{1}^{\mu} R_{\mu \nu}=k_{2}^{\mu} R_{\mu \nu}=0$. Some useful properties of the projectors are given in Appendix A. The virtual photon structure functions $W_{i}$ are functions of three invariants, i.e., $w$, 
$q^{2}\left(=-Q^{2}\right)$, and $p^{2}\left(=-P^{2}\right)$, and have no kinematical singularities. The subscripts " $T$ " and " $S$ " refer to the transverse and longitudinal photon, respectively. The structure functions with the superscript " $\tau$ " correspond to transitions with spinflip for each of the photons with total helicity conservation, while those with the superscript " $a$ " correspond to the $\mu \nu$ antisymmetric part of $W^{\mu \nu \rho \tau}$ and are measured, for example, through the two-photon processes in polarized $e^{+} e^{-}$collision experiments. These eight structure functions are related to the $s$-channel helicity amplitudes as follows [9]:

$$
\begin{aligned}
& W_{T T}=\frac{1}{2}[W(1,1 \mid 1,1)+W(1,-1 \mid 1,-1)], \\
& W_{S T}=W(0,1 \mid 0,1), \\
& W_{T S}=W(1,0 \mid 1,0), \quad W_{S S}=W(0,0 \mid 0,0), \\
& W_{T T}^{a}=\frac{1}{2}[W(1,1 \mid 1,1)-W(1,-1 \mid 1,-1)], \\
& W_{T T}^{\tau}=W(1,1 \mid-1,-1), \\
& W_{T S}^{\tau}=\frac{1}{2}[W(1,1 \mid 0,0)-W(1,0 \mid 0,-1)], \\
& W_{T S}^{\tau a}=\frac{1}{2}[W(1,1 \mid 0,0)+W(1,0 \mid 0,-1)] .
\end{aligned}
$$

Since the helicity-nonflip amplitudes are non-negative, the first four structure functions are positive definite and the last four are not. Due to the fact that the absorptive part $W^{\mu \nu \rho \tau}(p, q)$ is symmetric under the simultaneous interchange of $\{q, \mu, \nu\} \leftrightarrow\{p, \rho, \tau\}$, all the virtual photon structure functions, except $W_{S T}$ and $W_{T S}$, are symmetric under interchange of $p \leftrightarrow q$, while $W_{S T}\left(w, q^{2}, p^{2}\right)=W_{T S}\left(w, p^{2}, q^{2}\right)$. In terms of these structure functions, the positivity constraints (2.6)-(2.8) are rewritten as

$$
\begin{gathered}
\left|W_{T T}^{\tau}\right| \leqslant\left(W_{T T}+W_{T T}^{a}\right), \\
\left|W_{T S}^{\tau}+W_{T S}^{\tau a}\right| \leqslant \sqrt{\left(W_{T T}+W_{T T}^{a}\right) W_{S S}}, \\
\left|W_{T S}^{\tau}-W_{T S}^{\tau a}\right| \leqslant \sqrt{W_{T S} W_{S T}} .
\end{gathered}
$$

In fact, the following bounds,

$$
\left|W_{T T}^{\tau}\right| \leqslant 2 W_{T T}, \quad 2\left(W_{T S}^{\tau}\right)^{2} \leqslant 2 W_{S S} W_{T T}+W_{T S} W_{S T}
$$

were derived, some time ago, from the positiveness of the $\gamma \gamma$ cross section for arbitrary photon polarization [18]. Note that the constraints (2.13)-(2.15) which we have obtained are more stringent than the above ones [Eq. (2.16)].

\section{PARTON MODEL RESULTS}

For the real photon target, $P^{2}=0$, the number of independent structure functions or helicity amplitudes reduces to
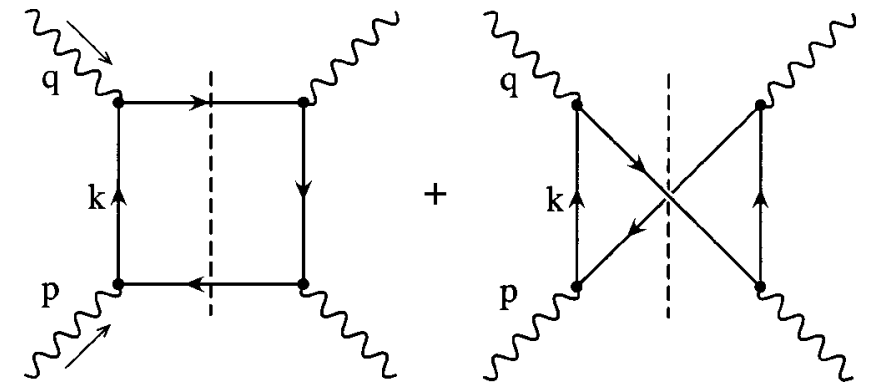

FIG. 2. The box diagrams in the parton model calculation.

four. They are $W_{T T}, W_{S T}, W_{T T}^{\tau}$, and $W_{T T}^{a}$, which are often referred to as

$$
\begin{aligned}
& W_{T T}=W_{1}^{\gamma}, \quad W_{S T}=\frac{1}{2 x} F_{L}^{\gamma}, \quad W_{T T}^{\tau}=2 W_{3}^{\gamma}, \\
& W_{T T}^{a}=W_{4}^{\gamma},
\end{aligned}
$$

and we have only one positivity constraint (2.13). In our previous paper [14] we have examined this constraint in the simple PM. Up to now most of our attention has been focused on the study of these four functions. In the case of the virtual photon target, $P^{2} \neq 0$, there appear four additional structure functions and we have derived three positivity constraints. But since we do not have much knowledge on the new photon structure functions, it is worthwhile, first, to investigate these functions in the simple PM and then to examine whether the three positivity constraints (2.13)-(2.15) actually hold.

We evaluate the box (massive quark-loop with a quark mass $m$ ) diagrams shown in Fig. 2. By applying the projectors, which were given in Eq. (2.10), to the box diagram contributions, we obtain the PM predictions for the eight virtual photon structure functions, $\left.W_{T T}\right|_{\mathrm{PM}},\left.W_{T T}^{a}\right|_{\mathrm{PM}}$, $\left.W_{T T}^{\tau}\right|_{\mathrm{PM}},\left.W_{S T}\right|_{\mathrm{PM}},\left.W_{T S}\right|_{\mathrm{PM}},\left.W_{S S}\right|_{\mathrm{PM}},\left.W_{T S}^{\tau}\right|_{\mathrm{PM}}$, and $\left.W_{T S}^{\tau a}\right|_{\mathrm{PM}}$. Their explicit expressions for the case $m \neq 0$ and $P^{2} \neq 0$ are given in Appendix B 2. The results are consistent with the cross sections for the $\gamma \gamma \rightarrow e^{+} e^{-}\left(\mu^{+} \mu^{-}\right)$process obtained by Budnev et al. [10] except for $\left.W_{T S}^{\tau a}\right|_{\mathrm{PM}} \cdot{ }^{1}$ Also the expressions of $\left.W_{T T}\right|_{\mathrm{PM}},\left.W_{S T}\right|_{\mathrm{PM}}$, and $\left.W_{S S}\right|_{\mathrm{PM}}$ are, respectively, in accord with those of $F_{T T}, F_{L T}$, and $F_{S S}$ given in Ref. [19].

We plot, in Figs. 3(a) and 3(b), these PM results for the eight photon structure functions as functions of $x$ $=Q^{2} /(2 p \cdot q)$. The vertical axes are in units ${ }^{2}$ of $(\alpha / 2 \pi) \delta_{\gamma}$, where $\delta_{\gamma}=3 \Sigma_{i}^{N_{f}} e_{i}^{4}$, with $N_{f}$ the number of active flavors. We have taken $P^{2} / Q^{2}=1 / 30$ and $m^{2} / Q^{2}=1 / 100$. The allowed $x$ region is $0 \leqslant x \leqslant x_{\max }$ with

\footnotetext{
${ }^{1}$ In the expression of $\tau_{T S}^{a}$, the last one in Eq. (E1) of Ref. [10] which corresponds to our $\left.W_{T S}^{\tau a}\right|_{\mathrm{PM}}$, the factor $\left[L+\left(q_{1} q_{2}\right) \Delta t / T\right]$ should read as $\left[L-\left(q_{1} q_{2}\right) \Delta t / T\right]$.

${ }^{2}$ Our definition of $W_{\mu \nu \rho \tau}$ and therefore of the photon structure functions, is such that they are proportional to $\alpha=e^{2} / 4 \pi$, and not to $\alpha^{2}$, in conformity with the nucleon case.
} 
(a)

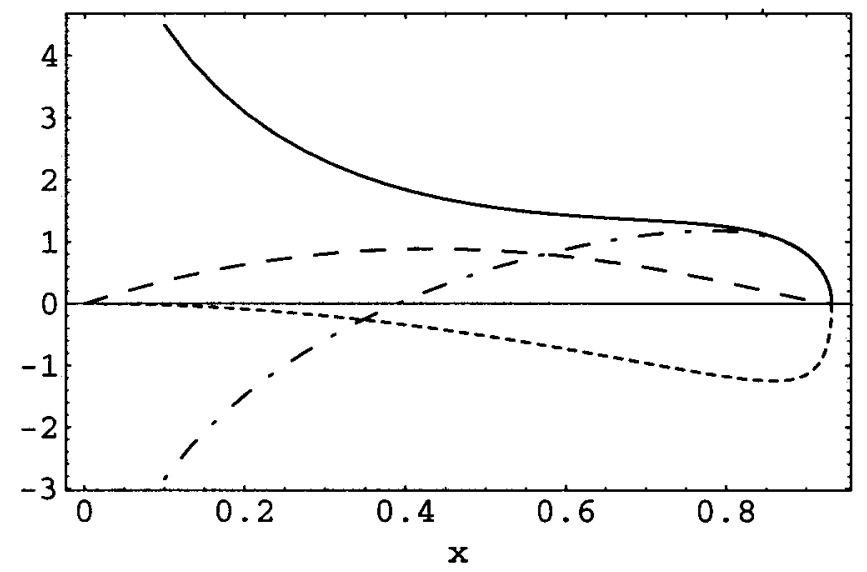

(b)

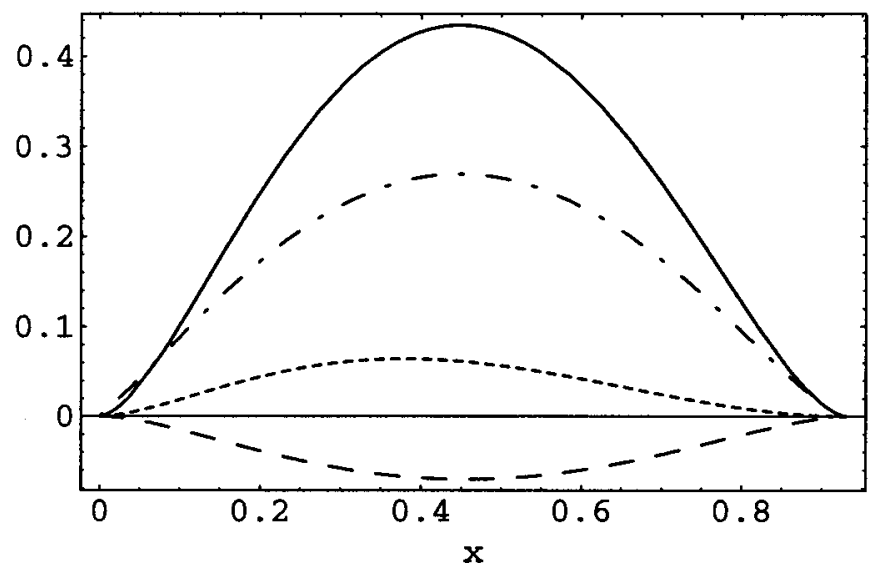

FIG. 3. The PM predictions versus $x$ for the eight virtual photon structure functions in units of $(\alpha / 2 \pi) \delta_{\gamma}$ for $P^{2} / Q^{2}=1 / 30$ and $m^{2} / Q^{2}=1 / 100$. (a) $\left.W_{T T}\right|_{\text {PM }}$ (solid line), $\left.W_{T T}^{a}\right|_{\text {PM }}$ (dash-dotted line), $\left.W_{S T}\right|_{\mathrm{PM}}$ (long-dashed line), and $\left.W_{T T}^{\tau}\right|_{\mathrm{PM}}$ (short-dashed line); (b) $\left.W_{T S}\right|_{\text {PM }}$ (solid line), $\left.W_{T S}^{\tau}\right|_{\text {PM }}$ (dash-dotted line), $\left.W_{T S}^{\tau a}\right|_{\text {PM }}$ (longdashed line), and $\left.W_{S S}\right|_{\mathrm{PM}}$ (short-dashed line).

$$
x_{\max }=1 /\left(1+\frac{P^{2}}{Q^{2}}+\frac{4 m^{2}}{Q^{2}}\right) .
$$

From Figs. 3(a) and 3(b), we see that the photon structure functions can be classified into three groups according to their magnitude: $\left.W_{T T}\right|_{\mathrm{PM}}$ and $\left.W_{T T}^{a}\right|_{\mathrm{PM}}$ are the first group, $\left.W_{S T}\right|_{\mathrm{PM}},\left.W_{T T}^{\tau}\right|_{\mathrm{PM}},\left.W_{T S}\right|_{\mathrm{PM}}$, and $\left.W_{T S}^{\tau}\right|_{\mathrm{PM}}$ are the second one, and $\left.W_{S S}\right|_{\mathrm{PM}}$ and $\left.W_{T S}^{\tau a}\right|_{\mathrm{PM}}$ are the third one. By comparison with $\left.W_{T T}\right|_{\mathrm{PM}}$ and $\left.W_{T T}^{a}\right|_{\mathrm{PM}}$ in the first group, $\left.W_{S S}\right|_{\mathrm{PM}}$ and $\left.W_{T S}^{\tau a}\right|_{\mathrm{PM}}$ are extremely small in magnitude. Also we see that the helicity-flip structure functions $\left.W_{T T}^{\tau}\right|_{\mathrm{PM}}$ and $\left.W_{T S}^{\tau}\right|_{\mathrm{PM}}$ are smaller in magnitude than the helicity-nonflip ones $\left.W_{T T}\right|_{\mathrm{PM}}$ and $\left.W_{T S}\right|_{\mathrm{PM}}$, respectively. We expect that these characteristics of the PM results will persist in the actual photon structure functions which would be obtained from future experiments.

The graphs in Figs. 3(a) and 3(b) show that all photon stucture functions tend to vanish as $x \rightarrow x_{\max }$ and this is the consequence of the kinematical constraint. For $x \rightarrow 0$, (a)

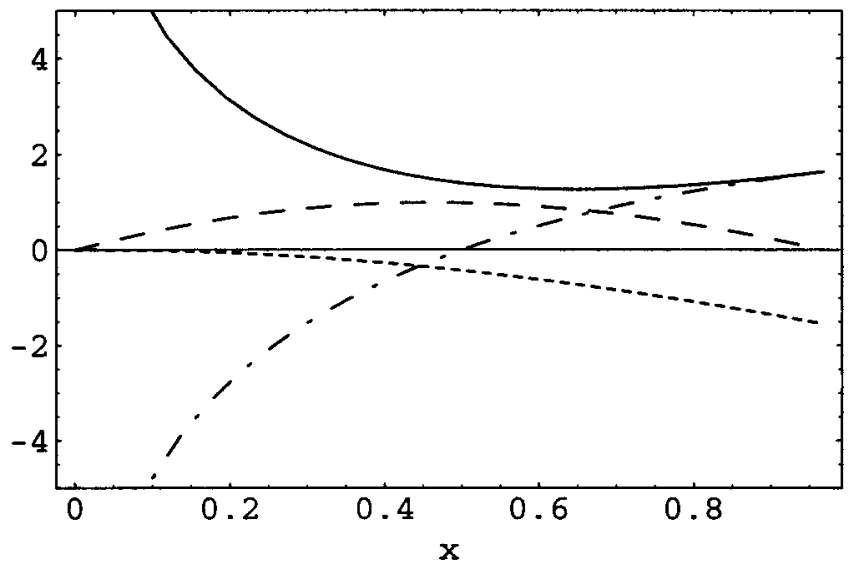

(b)

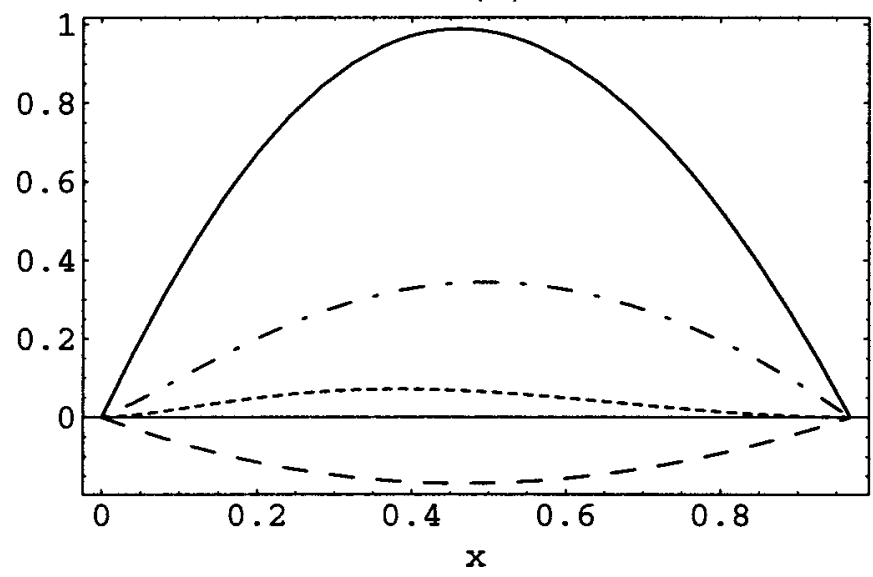

FIG. 4. The PM predictions versus $x$ for the eight virtual photon structure functions in units of $(\alpha / 2 \pi) \delta_{\gamma}$ for massless quark, $m$ $=0$, and $P^{2} / Q^{2}=1 / 30$. (a) $\left.W_{T T}\right|_{\mathrm{PM}}$ (solid line), $\left.W_{T T}^{a}\right|_{\mathrm{PM}}$ (dashdotted line), $\left.W_{S T}\right|_{\mathrm{PM}}$ (long-dashed line), and $\left.W_{T T}^{\tau}\right|_{\mathrm{PM}}$ (short-dashed line); (b) $\left.W_{T S}\right|_{\text {PM }}$ (solid line), $\left.W_{T S}^{\tau}\right|_{\text {PM }}$ (dash-dotted line), $\left.W_{T S}^{\tau a}\right|_{\text {PM }}$ (long-dashed line), and $\left.W_{S S}\right|_{P M}$ (short-dashed line). Note that $\left.W_{S T}\right|_{\mathrm{PM}}$ in (a) coincides with $\left.W_{T S}\right|_{\mathrm{PM}}$ in (b), as they should for $m$ $=0$.

$\left.W_{T T}\right|_{\mathrm{PM}}$ and $\left.W_{T T}^{a}\right|_{\mathrm{PM}}$ both diverge. The former diverges positively, and the later negatively. However, the sum remains finite since $\left(\left.W_{T T}\right|_{\mathrm{PM}}+\left.W_{T T}^{a}\right|_{\mathrm{PM}}\right) /\left((\alpha / 2 \pi) \delta_{\gamma}\right)$ as $x \rightarrow 0 \quad[\mathrm{see}$ Fig. 5(a) below]. The other structure functions vanish at $x$ $=0$.

It is interesting to note the clear difference, in the PM predictions for the photon structure functions, between the massive and the massless quark cases. We plot in Figs. 4(a) and 4 (b) the results for the massless quark case, $m=0$, with $P^{2} / Q^{2}=1 / 30$. Now three structure functions, $\left.W_{T T}\right|_{\mathrm{PM}}$, $\left.W_{T T}^{a}\right|_{\mathrm{PM}}$, and $\left.W_{T T}^{\tau}\right|_{\mathrm{PM}}$, do not vanish as $x \rightarrow x_{\max }$ and remain finite. For $x \rightarrow 0,\left.W_{T T}\right|_{\mathrm{PM}}$ and $\left.W_{T T}^{a}\right|_{\mathrm{PM}}$ both diverge again, but the sum tends to zero [see Fig. 6(a) below].

From the symmetry argument on the absorptive part $W^{\mu \nu \rho \tau}(p, q)$, we know that $W_{S T}$ and $W_{T S}$ switch into one another under the interchange of $p \leftrightarrow q$, namely, $W_{S T}\left(w, q^{2}, p^{2}\right)=W_{T S}\left(w, p^{2}, q^{2}\right)$. But this does not mean $W_{S T}=W_{T S}$. Indeed according to the PM results in the mas- 
(a)

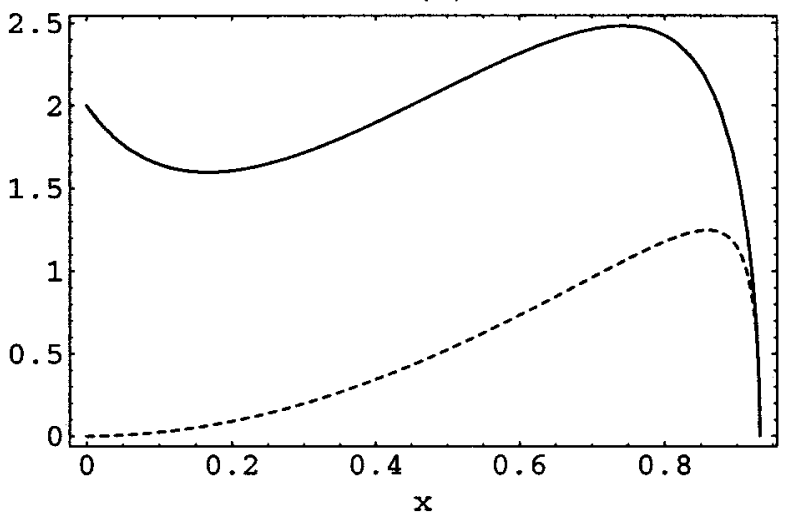

(b)

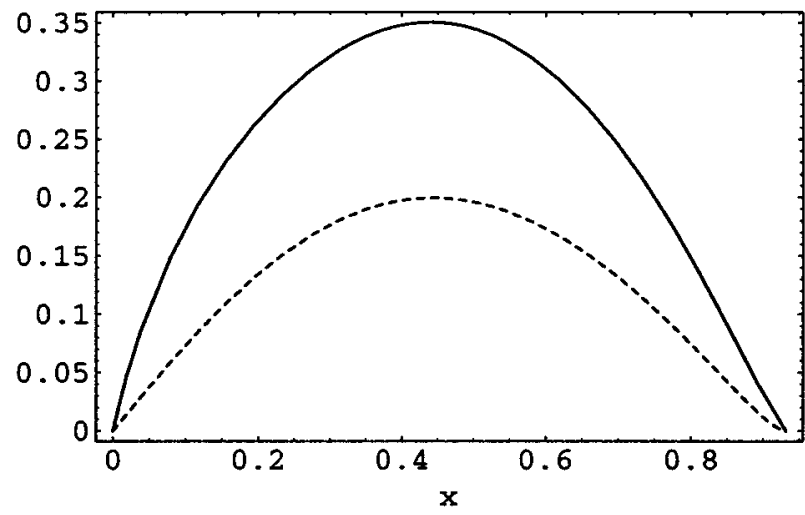

(c)

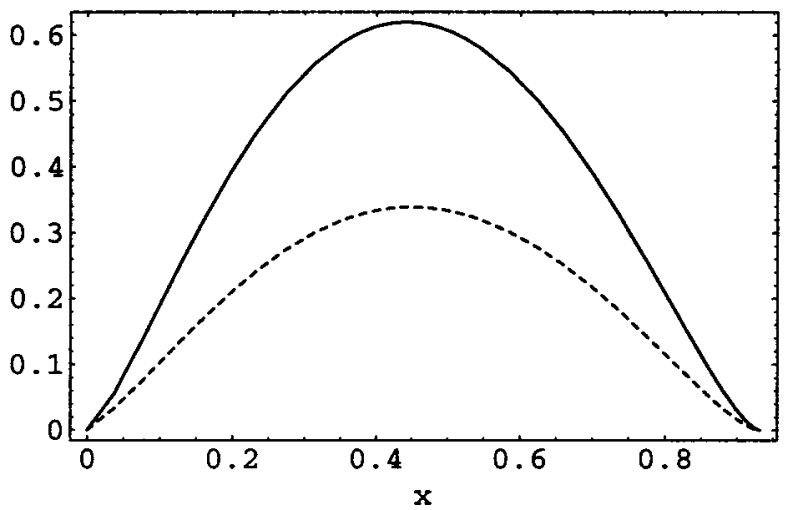

FIG. 5. The positivity constraints and the PM predictions versus $x$ for $P^{2} / Q^{2}=1 / 30$ and $m^{2} / Q^{2}=1 / 100$. The vertical axes are in units of $(\alpha / 2 \pi) \delta_{\gamma}$. (a) $\left(W_{T T}+W_{T T}^{a}\right)$ (solid line) and $\left|W_{T T}^{\tau}\right|$ (shortdashed line); (b) $\sqrt{\left(W_{T T}+W_{T T}^{a}\right) W_{S S}}$ (solid line) and $\left|W_{T S}^{\tau}+W_{T S}^{\tau a}\right|$ (short-dashed line); and (c) $\sqrt{W_{T S} W_{S T}}$ (solid line) and $\mid W_{T S}^{\tau}$ $-W_{T S}^{\tau a} \mid$ (short-dashed line).

sive quark case shown in Figs. 3(a) and 3(b), $\left.W_{S T}\right|_{\mathrm{PM}}$ and $\left.W_{T S}\right|_{\text {PM }}$ are different in magnitude and also have different $x$ dependences. However, we have found that in the limit $m$ $=0,\left.W_{S T}\right|_{\mathrm{PM}}$ coincides with $\left.W_{T S}\right|_{\mathrm{PM}}$ irrespectively to the values of $P^{2}$ and $Q^{2}$, which we believe is not a trivial result.

We plot in Fig. 5(a) the PM predictions versus $x$ of $\left(W_{T T}+W_{T T}^{a}\right)$ and $\left|W_{T T}^{\tau}\right|$, in Fig. 5(b) those of $\sqrt{\left(W_{T T}+W_{T T}^{a}\right) W_{S S}}$ and $\left|W_{T S}^{\tau}+W_{T S}^{\tau a}\right|$, and in Fig. 5(c) those of $\sqrt{W_{T S} W_{S T}}$ and $\left|W_{T S}^{\tau}-W_{T S}^{\tau a}\right|$, for the case $P^{2} / Q^{2}=1 / 30$ (a)

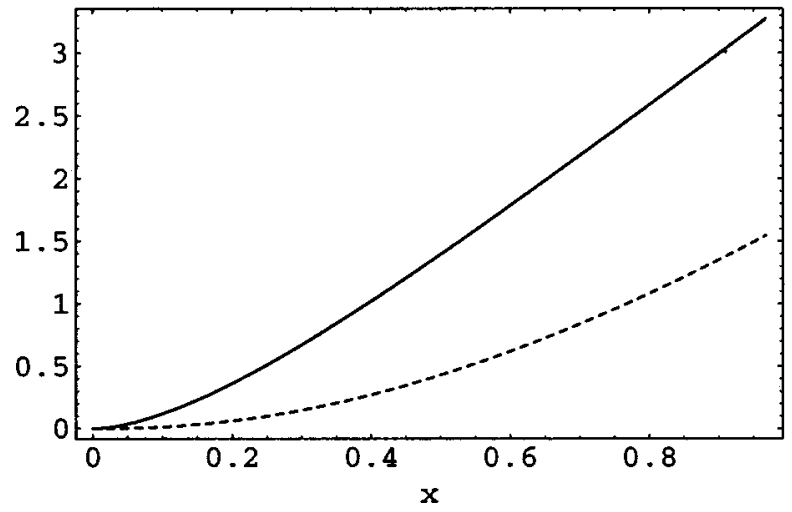

(b)

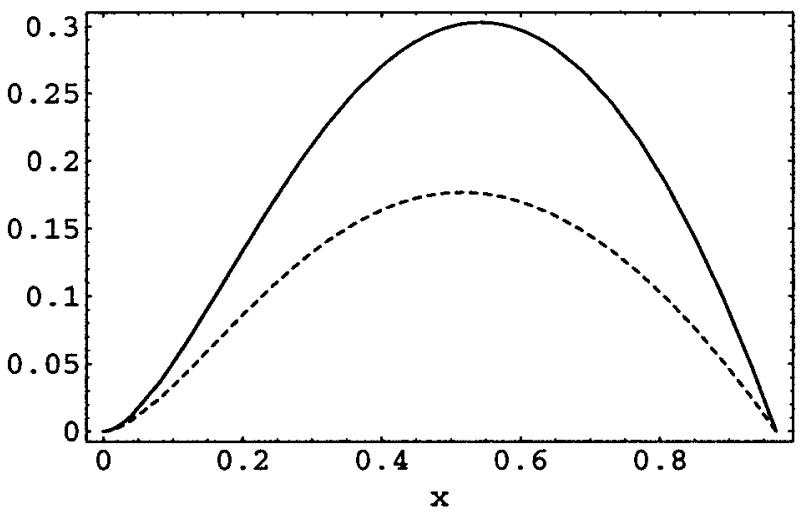

(c)

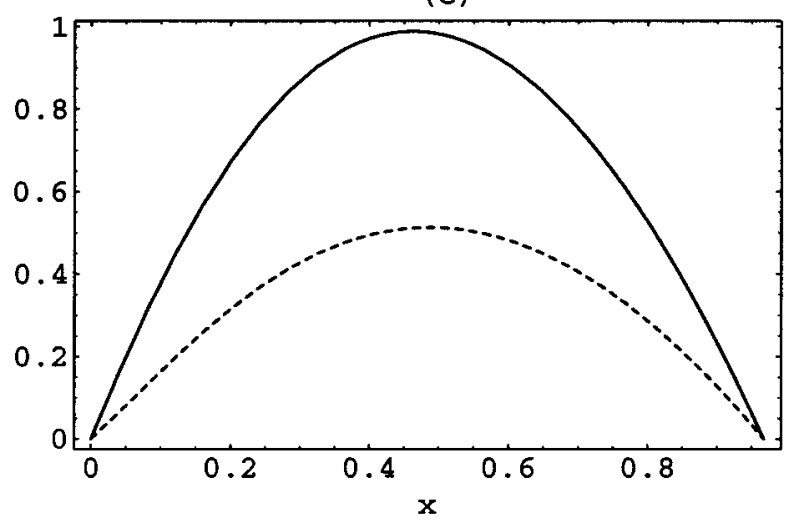

FIG. 6. Same as Fig. 5 for massless quark, $m=0$ and $P^{2} / Q^{2}$ $=1 / 30$.

and $m^{2} / Q^{2}=1 / 100$. For massless quark $m=0$, with $P^{2} / Q^{2}$ $=1 / 30$, similar plots are shown in Figs. 6(a)-6(c). In both cases we can see that the three positivity constraints (2.13)(2.15) are indeed satisfied for all the allowed $x$ region. However, as we have already mentioned above, the behaviors of the sum $\left(W_{T T}+W_{T T}^{a}\right)_{\mathrm{PM}}$ show a clear difference between the massive and the massless quark cases [see Figs. 5(a) and 6(a)]. For a massive quark, the sum reaches $2 \times\left((\alpha / 2 \pi) \delta_{\gamma}\right)$ as $x \rightarrow 0$ and the positivity constraint (2.13) is satisfied for all the allowed $x$ region with a wide margin. On the other hand, for a massless quark, it vanishes as $x$ $\rightarrow 0$ and the difference between $\left(W_{T T}+W_{T T}^{a}\right)_{\mathrm{PM}}$ and $\left|W_{T T}^{\tau}\right|_{\mathrm{PM}}$ reduces to zero. The fact that the sum $\left(W_{T T}\right.$ 
$\left.+W_{T T}^{a}\right)_{\mathrm{PM}}$ vanishes at $x=0$ in the case of the massless quark is explained as follows. The sum is related to a $s$-channel helicity amplitude of $\gamma-\gamma$ scattering, $W_{T T}+W_{T T}^{a}$ $=W(1,1 \mid 1,1)$. Now the limiting procedure $x=Q^{2} / 2 p \cdot q \rightarrow 0$ with the ratio $P^{2} / Q^{2}$ fixed is equivalent of taking $P^{2} \rightarrow 0$ and $Q^{2} \rightarrow 0$ and keeping $2 p \cdot q$ finite. So the situation at $x=0$ is the same as if we were dealing with the two real photon scattering process, $\gamma+\gamma \rightarrow q+\bar{q}$. Since chirality coincides with helicity for the massless quark and the electromagnetic interaction preserves the quark chirality, it is known that the amplitude for the two real photons with the same helicity annihilating into a massless quark pair vanishes identically [20].

\section{PERTURBATIVE QCD}

Now we switch on the QCD coupling. The photon structure functions have been studied by PQCD for many years [2]. Especially, in the kinematic region,

$$
\Lambda^{2} \ll P^{2} \ll Q^{2}
$$

where the mass squared of the target photon $\left(P^{2}\right)$ is much larger than the QCD scale parameter $\left(\Lambda^{2}\right)$, some of the photon structure functions are predictable in PQCD entirely up to the NLO. This is due to the fact that, in this kinematical region, the hadronic components of the photon (in other words, the photon matrix elements of hadronic operators) can be calculated perturbatively. Indeed, the virtual photon structure functions, such as unpolarized $F_{2}^{\gamma}\left(x, Q^{2}, P^{2}\right)$ and $F_{L}^{\gamma}\left(x, Q^{2}, P^{2}\right)[21]$ and polarized $g_{1}^{\gamma}\left(x, Q^{2}, P^{2}\right)$ [6], were studied up to the NLO in the above kinematic region. Here the virtual photon structure functions $F_{2}^{\gamma}, F_{L}^{\gamma}$, and $g_{1}^{\gamma}$ are related to the ones introduced by $\mathrm{BCG}$ in Eq. (2.9) as follows: ${ }^{3}$

$$
\begin{aligned}
& F_{1}^{\gamma}\left(x, Q^{2}, P^{2}\right)=2\left[W_{T T}-\frac{1}{2} W_{T S}\right] \\
& F_{2}^{\gamma}\left(x, Q^{2}, P^{2}\right)=\frac{2 x}{\widetilde{\beta}^{2}}\left[W_{T T}+W_{S T}-\frac{1}{2} W_{S S}-\frac{1}{2} W_{T S}\right], \\
& F_{L}^{\gamma}\left(x, Q^{2}, P^{2}\right)=F_{2}^{\gamma}-x F_{1}^{\gamma}, \\
& g_{1}^{\gamma}\left(x, Q^{2}, P^{2}\right)=\frac{2}{\widetilde{\beta}^{2}}\left[W_{T T}^{a}-\frac{\left(P^{2} Q^{2}\right)^{1 / 2}}{w} W_{T S}^{\tau a}\right]
\end{aligned}
$$

with $\widetilde{\beta}=\left(1-P^{2} Q^{2} / w^{2}\right)^{1 / 2}$.

Since the tensor $W^{\mu \nu \rho \tau}(p, q)$ in Eq. (2.9) is regular as $p^{2} \rightarrow 0$, while the projectors $P_{T S}$ and $P_{T S}^{\tau a}$ are singular as $p^{2}$ $\rightarrow 0$ and behave as $1 / p^{2}$ and $1 / \sqrt{-p^{2}}$, respectively, we expect $W_{T S} \propto P^{2} / Q^{2}$ and $W_{T S}^{\tau a} \propto \sqrt{P^{2} / Q^{2}}$. Then, in the kinematic

\footnotetext{
${ }^{3}$ We follow Nisius [22] for the definition of $F_{1}^{\gamma}, F_{2}^{\gamma}$, and $F_{L}^{\gamma}$ apart from $F_{1}^{\gamma}$ being different from the one of Nisius by a factor of 2 . For other definitions of $F_{1}^{\gamma}, F_{2}^{\gamma}$, and $F_{L}^{\gamma}$, see Refs. [7,23].
}

region (4.1), $\widetilde{\beta} \approx 1$, and we can neglect the contributions of $W_{T S}$ and $W_{T S}^{\tau a}$ as compared with $W_{T T}$ and $W_{T T}^{a}$, respectively. As a result we have

$$
\begin{aligned}
W_{T T}\left(x, Q^{2}, P^{2}\right) & \approx \frac{1}{2} F_{1}^{\gamma}\left(x, Q^{2}, P^{2}\right) \\
& =\frac{1}{2 x}\left\{F_{2}^{\gamma}\left(x, Q^{2}, P^{2}\right)-F_{L}^{\gamma}\left(x, Q^{2}, P^{2}\right)\right\}, \\
W_{T T}^{a}\left(x, Q^{2}, P^{2}\right) & \approx \frac{1}{2} g_{1}^{\gamma}\left(x, Q^{2}, P^{2}\right) .
\end{aligned}
$$

The positivity constraint (2.13) is now rewritten as

$\left|W_{T T}^{\tau}\left(x, Q^{2}, P^{2}\right)\right| \lesssim \frac{1}{2}\left[F_{1}^{\gamma}\left(x, Q^{2}, P^{2}\right)+g_{1}^{\gamma}\left(x, Q^{2}, P^{2}\right)\right]$,

and it is interesting to see if this inequality is satisfied by the PQCD results. For $F_{2}^{\gamma}\left(x, Q^{2}, P^{2}\right)$ and $F_{L}^{\gamma}\left(x, Q^{2}, P^{2}\right)$, we can take the results from Ref. [21] and for $g_{1}^{\gamma}\left(x, Q^{2}, P^{2}\right)$ we use Ref. [6]. Actually the PQCD results for $F_{1}^{\gamma}$ and $g_{1}^{\gamma}$ are given in the form of Mellin moments, and we need to perform the inverse Mellin transformation in order to express them as functions of $x$. The formula for the $n$th moment of $F_{1}^{\gamma}$ up to the NLO is summarized in Appendix C. After the inverse Mellin transformation, $\frac{1}{2}\left(F_{1}^{\gamma}+g_{1}^{\gamma}\right)$ is expressed in the form as

$$
\frac{1}{2}\left(F_{1}^{\gamma}+g_{1}^{\gamma}\right)=\frac{\alpha}{2 \pi} \delta_{\gamma}\left[a(x) \ln \frac{Q^{2}}{\Lambda^{2}}+b(x)+\mathcal{O}\left[\alpha_{s}\left(Q^{2}\right)\right],\right.
$$

where $\Lambda^{2}$ is the QCD scale and $\alpha_{s}\left(Q^{2}\right)$ is the QCD running coupling constant.

The virtual photon structure function $W_{T T}^{\tau}\left(x, Q^{2}, P^{2}\right)$ $\left(=2 W_{3}^{\gamma}\right)$ is expected to be given by the same expression as the PM result up to $\mathcal{O}\left[1 / \ln \left(Q^{2} / \Lambda^{2}\right)\right]$, since there exist no twist-2 quark operators contributing to $W_{T T}^{\tau}$. So we take, in the leading order (LO) [24,25],

$$
W_{T T}^{\tau}\left(x, Q^{2}, P^{2}\right)=\frac{\alpha}{2 \pi} \delta_{\gamma}\left\{\left(-2 x^{2}\right)+\mathcal{O}\left[\alpha_{s}\left(Q^{2}\right)\right]\right\}
$$

where the first term is derived from $\left.W_{T T}^{\tau}\right|_{\mathrm{PM}}$ given in Eq. (B5), ignoring the power corrections of $m^{2} / Q^{2}$ and $P^{2} / Q^{2}$.

Now we plot, in Fig. 7, the NLO PQCD result of $\frac{1}{2}\left(F_{1}^{\gamma}\right.$ $\left.+g_{1}^{\gamma}\right)$ and the LO result of $\left|W_{T T}^{\tau}\right|$ as functions of $x$ for the case $P^{2} / Q^{2}=1 / 30$ with the number of active flavors, $N_{f}$ $=3$. We find that the inequality (4.4) is satisfied for almost all the allowed $x$ region except near $x_{\max }=Q^{2} /\left(Q^{2}+P^{2}\right)$ $\left(\approx 0.968\right.$ for $\left.P^{2} / Q^{2}=1 / 30\right)$. The violation of the inequality near $x_{\max }$ is explained as follows. We observe that the graph of $\frac{1}{2}\left(F_{1}^{\gamma}+g_{1}^{\gamma}\right)$ falls rapidly as $x \rightarrow x_{\max }$. In the language of the QCD improved parton model, this is due to total momentum conservation of all partons in the photon. In fact, the moments of both $F_{1}^{\gamma}$ and $g_{1}^{\gamma}$ in the LO behave as $1 /(n \ln n)$ for large $n$ and thus in $x$ space they vanish like $-1 / \ln (1$ $-x)$ as $x \rightarrow 1$. The NLO QCD corrections further suppress $F_{1}^{\gamma}$ 


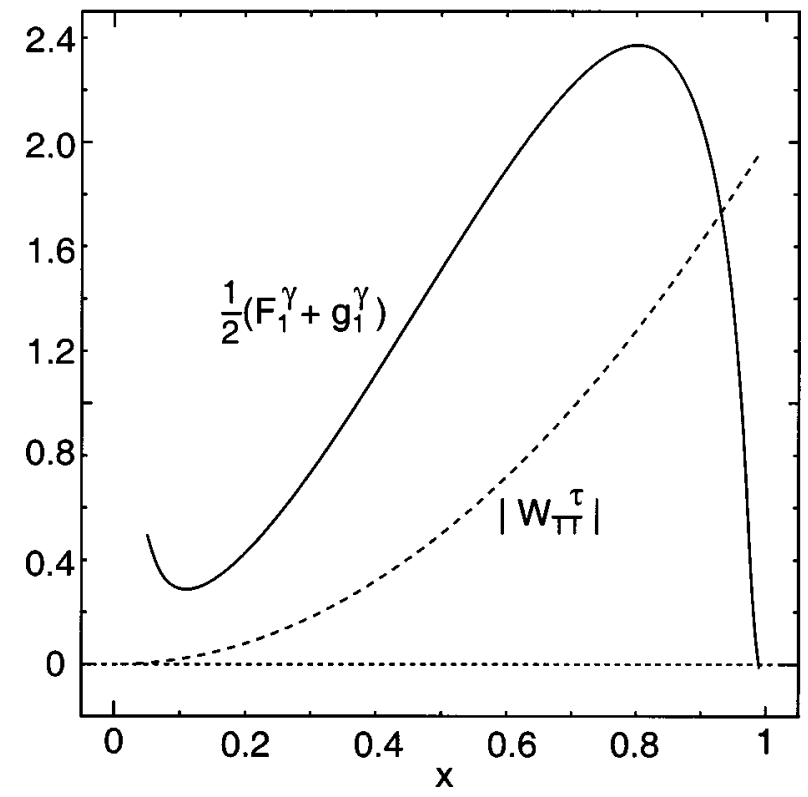

FIG. 7. The positivity constraint and the PQCD prediction. $\frac{1}{2}\left(F_{1}^{\gamma}+g_{1}^{\gamma}\right)$ (solid line) and $\left|W_{T T}^{\tau}\right|$ (short-dashed line) in units of $(\alpha / 2 \pi) \delta_{\gamma}$, for $Q^{2}=30 \mathrm{GeV}^{2}, P^{2}=1 \mathrm{GeV}^{2}$ with $\Lambda=0.2 \mathrm{GeV}$ and $N_{f}=3$.

and $g_{1}^{\gamma}$ at large $x$. On the other hand, the LO QCD prediction of $W_{T T}^{\tau}$ is the same as the massless quark PM result, with the power corrections of $P^{2} / Q^{2}$ being neglected. Thus $\left|W_{T T}^{\tau}\right|$ in Fig. 7 increases monotonically as a function of $x^{2}$ and the violation of the inequality (4.4) occurred near $x_{\max }$.

However, the physical $W_{T T}^{\tau}$ should vanish as $x \rightarrow x_{\max }$ due to kinematical constraints. The momentum conservation of partons is not applicable here since quark partons in the photon do not contribute to $W_{T T}^{\tau}$ in the LO, in other words, there exist no twist-2 quark operators relevant to $W_{T T}^{\tau}$ [24]. This urges the necessity of introducing the quark mass effects to the calculation of the photon coefficient function. (Remember that $\left.W_{T T}^{\tau}\right|_{\mathrm{PM}}$ in the massive PM vanishes as $x \rightarrow x_{\max }$.)

Except for large and small $x$, we find that the PQCD prediction for $\frac{1}{2}\left(F_{1}^{\gamma}+g_{1}^{\gamma}\right)$ appears to be similar to the massless quark PM result for $\left(W_{T T}+W_{T T}^{a}\right)$. In fact, for moderate $x$, $0.2 \leqslant x \leqslant 0.7$, the graph $\frac{1}{2}\left(F_{1}^{\gamma}+g_{1}^{\gamma}\right)$ resembles closely the massless quark result $\left(W_{T T}+W_{T T}^{a}\right)_{\mathrm{PM}}$ in Fig. 6(a) in shape and magnitude. As $x \rightarrow 0$, we find that the sum $\frac{1}{2}\left(F_{1}^{\gamma}+g_{1}^{\gamma}\right)$ starts to increase.

\section{CONCLUSION}

To summarize we have investigated the three positivity constraints for the virtual photon structure functions which could be studied in future $e p$ and $e^{+} e^{-}$colliders. In particular the virtual photon structure can be measured from the double-tagged $e^{+} e^{-}$reactions and also from the dijet events in deep inelastic ep collisions.

By evaluating the quark box-diagrams, we obtained the eight virtual photon structure functions in the PM both for a massive and a massless quark. It has turned out that there exists a clear difference both in $x$-dependence and in magnitude between the massive and massless quark PM predictions. We have found that the three constraints indeed hold for the PM computation of both massive and massless quark cases. In the kinematic region, $\Lambda^{2} \ll P^{2} \ll Q^{2}$, the NLO QCD results for $F_{1}^{\gamma}$ and $g_{1}^{\gamma}$ and the LO result for $W_{T T}^{\tau}$ satisfy the constraint among these three structure functions for most of the allowed $x$ region except for the region very near $x_{\max }$.

We expect that these bounds will provide useful constraints for studying the yet unknown polarized and unpolarized virtual photon structure functions.

\section{ACKNOWLEDGMENTS}

We thank Werner Vogelsang and Hideshi Baba for useful discussions. This work was partially supported by the Grantin-Aid from the Japan Ministry of Education and Science, No. (C)(2)-12640266.

\section{APPENDIX A: PROJECTORS FOR THE VIRTUAL PHOTON STRUCTURE FUNCTIONS}

The projectors $P_{i}$ 's are defined in Eq. (2.10). $\left(P_{T T}^{a}\right)^{\mu \nu \rho \tau}$ and $\left(P_{T S}^{\tau a}\right)^{\mu \nu \rho \tau}$ are antisymmetric under the interchange of $\mu \leftrightarrow \nu$ and $\rho \leftrightarrow \tau$, while other projectors are symmetric. Since $R_{\mu \nu}, k_{1}$, and $k_{2}$ have the following properties:

$$
\begin{aligned}
& R_{\mu \nu} q^{\mu}=0, \quad R_{\mu \nu} p^{\mu}=0, \quad R_{\mu \rho} R_{\nu}^{\rho}=-R_{\mu \nu}, \quad R_{\mu \nu} R^{\mu \nu}=2, \\
& k_{1}^{\mu} R_{\mu \nu}=0, \quad k_{2}^{\mu} R_{\mu \nu}=0, \quad k_{1}^{2}=k_{2}^{2}=1,
\end{aligned}
$$

we find

$$
\begin{aligned}
\left(P_{i}\right)^{\mu \nu \rho \tau}\left(P_{j}\right)_{\mu \nu \rho \tau}=0 & \text { for } \quad i \neq j, \\
\left(P_{T T}\right)^{\mu \nu \rho \tau}\left(P_{T T}\right)_{\mu \nu \rho \tau}=4, & \left(P_{T T}^{a}\right)^{\mu \nu \rho \tau}\left(P_{T T}^{a}\right)_{\mu \nu \rho \tau}=4, \\
\left(P_{T T}^{\tau}\right)^{\mu \nu \rho \tau}\left(P_{T T}^{\tau}\right)_{\mu \nu \rho \tau}=2, & \left(P_{S T}\right)^{\mu \nu \rho \tau}\left(P_{S T}\right)_{\mu \nu \rho \tau}=2, \\
\left(P_{T S}\right)^{\mu \nu \rho \tau}\left(P_{T S}\right)_{\mu \nu \rho \tau}=2, & \left(P_{S S}\right)^{\mu \nu \rho \tau}\left(P_{S S}\right)_{\mu \nu \rho \tau}=1, \\
\left(P_{T S}^{\tau}\right)^{\mu \nu \rho \tau}\left(P_{T S}^{\tau}\right)_{\mu \nu \rho \tau}=8, & \left(P_{T S}^{\tau a}\right)^{\mu \nu \rho \tau}\left(P_{T S}^{\tau a}\right)_{\mu \nu \rho \tau}=8 .
\end{aligned}
$$

\section{APPENDIX B: VIRTUAL PHOTON STRUCTURE FUNCTIONS IN PARTON MODEL}

\section{Parameters}

$$
\begin{aligned}
& x=\frac{Q^{2}}{2 p \cdot q}, \quad \delta_{\gamma}=3 \sum_{i=1}^{N_{f}} e_{i}^{4}, \\
& \widetilde{\beta}=\sqrt{1-\frac{P^{2} Q^{2}}{(p \cdot q)^{2}}}, \quad \beta=\sqrt{1-\frac{4 m^{2}}{(p+q)^{2}}}, \\
& L=\ln \frac{1+\beta \widetilde{\beta}}{1-\beta \widetilde{\beta}},
\end{aligned}
$$

where $N_{f}$ is the number of the active flavors and $m$ is the quark mass. 


\section{Structure functions in PM}

$$
\begin{aligned}
\left.W_{T T}\right|_{\mathrm{PM}}= & \frac{\alpha}{2 \pi} \delta_{\gamma}\left[L \left\{-\frac{8 x^{2}}{\widetilde{\beta}} \frac{m^{4}}{Q^{4}}+\frac{\widetilde{\beta}^{2}-(1-2 x)^{2}}{\widetilde{\beta}^{3}} \frac{m^{2}}{Q^{2}}+\frac{1}{8 \widetilde{\beta}^{5}}\left[\left(1-\widetilde{\beta}^{2}\right)\left(\widetilde{\beta}^{4}+3\right)-8 x\left\{\widetilde{\beta}^{4}-2 \widetilde{\beta}^{2}+3\right\}\right] \frac{P^{2}}{Q^{2}}\right.\right. \\
& \left.+\frac{1}{4 \widetilde{\beta}^{5}}\left[-\widetilde{\beta}^{6}+\left(2 x^{2}-4 x+7\right) \widetilde{\beta}^{4}+(8 x-11) \widetilde{\beta}^{2}+3\left(2 x^{2}-4 x+3\right)\right]\right\}+\frac{\beta}{1-\beta^{2} \widetilde{\beta}^{2}}\left\{-16 x^{2} \frac{m^{4}}{Q^{4}}\right. \\
& +\frac{2\left[(1-2 x)^{2}+(4 x-1) \widetilde{\beta}^{2}\right]}{\widetilde{\beta}^{2}} \frac{m^{2}}{Q^{2}}+\frac{1}{4 \widetilde{\beta}^{4}}\left[\left(1-\widetilde{\beta}^{2}\right)\left(\widetilde{\beta}^{4}+2 \beta^{2} \widetilde{\beta}^{2}-3\right)+8 x\left\{\beta^{2} \widetilde{\beta}^{4}-2\left(\beta^{2}+1\right) \widetilde{\beta}^{2}+3\right\}\right] \frac{P^{2}}{Q^{2}} \\
& \left.\left.+\frac{1}{2 \widetilde{\beta}^{4}}\left[\left(2 \beta^{2}+1\right) \widetilde{\beta}^{6}+\left\{2 x^{2}+4 \beta^{2} x-6 \beta^{2}-5\right\} \widetilde{\beta}^{4}+\left\{4 \beta^{2} x^{2}-8\left(\beta^{2}+1\right) x+6 \beta^{2}+11\right\} \widetilde{\beta}^{2}-3\left(2 x^{2}-4 x+3\right)\right]\right\}\right],
\end{aligned}
$$

$$
\begin{aligned}
\left.W_{T T}^{a}\right|_{\mathrm{PM}}= & \frac{\alpha}{2 \pi} \delta_{\gamma}\left[L \frac{1}{\widetilde{\beta}^{3}}\left\{2 x \frac{P^{2}}{Q^{2}}+\left(\widetilde{\beta}^{2}+2 x-2\right)\right\}+\frac{\beta}{1-\beta^{2} \widetilde{\beta}^{2}}\left\{8 x \frac{m^{2}}{Q^{2}}+\frac{2 x\left\{\left(\beta^{2}+1\right) \widetilde{\beta}^{2}-2\right\}}{\widetilde{\beta}^{2}} \frac{P^{2}}{Q^{2}}\right.\right. \\
& \left.\left.+\frac{\left(\widetilde{\beta}^{2}+2 x-2\right)\left\{\left(\beta^{2}+1\right) \widetilde{\beta}^{2}-2\right\}}{\widetilde{\beta}^{2}}\right\}\right],
\end{aligned}
$$

$$
\begin{aligned}
\left.W_{T T}^{\tau}\right|_{\mathrm{PM}}= & \frac{\alpha}{2 \pi} \delta_{\gamma}\left[2 L \left\{-\frac{4 x^{2}}{\widetilde{\beta}} \frac{m^{4}}{Q^{4}}+\frac{\widetilde{\beta}^{4}-4 x(x+1) \widetilde{\beta}^{2}-(1-2 x)^{2}}{2 \widetilde{\beta}^{3}} \frac{m^{2}}{Q^{2}}\right.\right. \\
& \left.+\frac{1-\widetilde{\beta}^{2}}{16 \widetilde{\beta}^{5}}\left[\left(1-\widetilde{\beta}^{2}\right)\left(3+\widetilde{\beta}^{2}\right)+8 x\left(\widetilde{\beta}^{2}-3\right)\right] \frac{P^{2}}{Q^{2}}+\frac{1-\widetilde{\beta}^{2}}{8 \widetilde{\beta}^{5}}\left[3 \widetilde{\beta}^{4}+2\left(x^{2}+2 x-4\right) \widetilde{\beta}^{2}+3\left(2 x^{2}-4 x+3\right)\right]\right\} \\
& +\frac{2 \beta}{1-\beta^{2} \widetilde{\beta}^{2}}\left\{-8 x^{2} \frac{m^{4}}{Q^{4}}+\frac{\left(1-\widetilde{\beta}^{2}\right)\left[(1-2 x)^{2}-\widetilde{\beta}^{2}\right]}{m^{2}} \frac{1-\widetilde{\beta}^{2}}{8 \widetilde{\beta}^{2}}\left[\widetilde{\beta}^{4}-2\left(\beta^{2}+1\right) \widetilde{\beta}^{2}+3\right.\right. \\
& \left.+8 x\left\{\left(2 \beta^{2}+1\right) \widetilde{\beta}^{2}-3\right\}\right] \frac{P^{2}}{Q^{2}}+\frac{1}{4 \widetilde{\beta}^{4}}\left[\left(4 \beta^{2}+1\right) \widetilde{\beta}^{6}-\left\{2 x^{2}-4\left(2 \beta^{2}+1\right) x+10 \beta^{2}+9\right\} \widetilde{\beta}^{4}\right. \\
& \left.\left.\left.+\left\{4\left(\beta^{2}+1\right) x^{2}-8\left(\beta^{2}+2\right) x+6 \beta^{2}+17\right\} \widetilde{\beta}^{2}-3\left(2 x^{2}-4 x+3\right)\right]\right\}\right]
\end{aligned}
$$

$$
\begin{aligned}
\left.W_{S T}\right|_{\mathrm{PM}}= & \frac{\alpha}{2 \pi} \delta_{\gamma}\left[L \left\{-\frac{\widetilde{\beta}^{4}+\left(4 x^{2}+4 x-2\right) \widetilde{\beta}^{2}+(1-2 x)^{2}}{\widetilde{\beta}^{3}} \frac{m^{2}}{Q^{2}}+\frac{1-\widetilde{\beta}^{2}}{4 \widetilde{\beta}^{5}}\left[\left(1-\widetilde{\beta}^{2}\right)\left(3+\widetilde{\beta}^{2}\right)-24 x\right] \frac{P^{2}}{Q^{2}}\right.\right. \\
& \left.-\frac{1-\widetilde{\beta}^{2}}{2 \widetilde{\beta}^{5}}\left[\widetilde{\beta}^{4}-2\left(x^{2}-2\right) \widetilde{\beta}^{2}-3\left(2 x^{2}-4 x+3\right)\right]\right\}+\frac{\beta}{1-\beta^{2} \widetilde{\beta}^{2}}\left\{\frac{2\left(1-\widetilde{\beta}^{2}\right)\left[(1-2 x)^{2}-\widetilde{\beta}^{2}\right]}{\widetilde{\beta}^{2}} \frac{\widetilde{\beta}^{2}}{Q^{2}}\right. \\
& +\frac{1-\widetilde{\beta}^{2}}{2 \widetilde{\beta}^{4}}\left[\left(1-\widetilde{\beta}^{2}\right)\left\{\left(2 \beta^{2}-1\right) \widetilde{\beta}^{2}-3\right\}-8 x\left(2 \beta^{2} \widetilde{\beta}^{2}-3\right)\right] \frac{P^{2}}{Q^{2}}+\frac{1}{\widetilde{\beta}^{4}}\left[\beta^{2} \widetilde{\beta}^{6}-\left\{2 x^{2}-4\left(\beta^{2}+1\right) x\right.\right. \\
& \left.\left.\left.\left.+7 \beta^{2}+4\right\} \widetilde{\beta}^{4}+\left\{4\left(\beta^{2}+1\right) x^{2}-4\left(2 \beta^{2}+3\right) x+6 \beta^{2}+13\right\} \widetilde{\beta}^{2}-3\left(2 x^{2}-4 x+3\right)\right]\right\}\right]
\end{aligned}
$$




$$
\begin{aligned}
& \left.W_{T S}\right|_{\mathrm{PM}}=\frac{\alpha}{2 \pi} \delta_{\gamma}\left[L ( 1 - \widetilde { \beta } ^ { 2 } ) \left\{-\frac{4 x^{2}-4 x+\widetilde{\beta}^{2}+1}{\widetilde{\beta}^{3}} \frac{m^{2}}{Q^{2}}+\frac{1}{4 \widetilde{\beta}^{5}}\left[\left(1-\widetilde{\beta}^{2}\right)\left(3+\widetilde{\beta}^{2}\right)-24 x\right] \frac{P^{2}}{Q^{2}}\right.\right. \\
& \left.-\frac{1}{2 \widetilde{\beta}^{5}}\left[\widetilde{\beta}^{4}-2\left(x^{2}-2\right) \widetilde{\beta}^{2}-3\left(2 x^{2}-4 x+3\right)\right]\right\}+\frac{\beta}{1-\beta^{2} \widetilde{\beta}^{2}}\left\{\frac{2\left(1-\widetilde{\beta}^{2}\right)\left[(1-2 x)^{2}-\widetilde{\beta}^{2}\right]}{\widetilde{\beta}^{2}} \frac{m^{2}}{Q^{2}}\right. \\
& +\frac{1}{2 \widetilde{\beta}^{4}}\left[-\left(1-\widetilde{\beta}^{2}\right)\left\{\widetilde{\beta}^{4}-2\left(\beta^{2}+1\right) \widetilde{\beta}^{2}+3\right\}+8 x\left\{\left(\beta^{2}+1\right) \widetilde{\beta}^{4}-\left(2 \beta^{2}+3\right) \widetilde{\beta}^{2}+3\right\}\right] \frac{P^{2}}{Q^{2}} \\
& \left.\left.-\frac{1-\widetilde{\beta}^{2}}{\widetilde{\beta}^{4}}\left[\beta^{2} \widetilde{\beta}^{4}-2\left\{\left(2 \beta^{2}-1\right) x^{2}-4 \beta^{2} x+3 \beta^{2}+2\right\} \widetilde{\beta}^{2}+3\left(2 x^{2}-4 x+3\right)\right]\right\}\right], \\
& \left.W_{S S}\right|_{\mathrm{PM}}=\frac{\alpha}{2 \pi} \delta_{\gamma}\left[L \frac{\left(1-\widetilde{\beta}^{2}\right)\left(3-\widetilde{\beta}^{2}\right)}{2 \widetilde{\beta}^{5}}-\frac{\beta\left(1-\widetilde{\beta}^{2}\right)\left\{3-\left(2 \beta^{2}+1\right) \widetilde{\beta}^{2}\right\}}{\left(1-\beta^{2} \widetilde{\beta}^{2}\right) \widetilde{\beta}^{4}}\right]\left\{\left[\left(1-\widetilde{\beta}^{2}\right)-8 x\right] \frac{P^{2}}{Q^{2}}+2\left(2 x^{2}-4 x+3-\widetilde{\beta}^{2}\right)\right\}, \\
& \left.W_{T S}^{\tau}\right|_{\mathrm{PM}}=-\frac{\alpha}{2 \pi} \delta_{\gamma}\left[L \frac { \sqrt { 1 - \widetilde { \beta } ^ { 2 } } } { \widetilde { \beta } ^ { 5 } } \left\{\widetilde{\beta}^{2}\left[\widetilde{\beta}^{2}-(1-2 x)^{2}\right] \frac{m^{2}}{Q^{2}}+\left[\frac{1}{4}\left(1-\widetilde{\beta}^{2}\right)\left(3-\widetilde{\beta}^{2}\right)+2 x\left(2 \widetilde{\beta}^{2}-3\right)\right] \frac{P^{2}}{Q^{2}}\right.\right. \\
& \left.+\frac{1}{2}\left[\widetilde{\beta}^{4}-2\left(x^{2}-4 x+5\right) \widetilde{\beta}^{2}+3\left(2 x^{2}-4 x+3\right)\right]\right\}+\frac{\beta \sqrt{1-\widetilde{\beta}^{2}}}{\widetilde{\beta}^{4}\left(1-\beta^{2} \widetilde{\beta}^{2}\right)}\left\{2 \widetilde{\beta}^{2}(2 x-1)\left(2 x-1+\widetilde{\beta}^{2}\right) \frac{m^{2}}{Q^{2}}\right. \\
& +\left[\frac{1}{2}\left(1-\widetilde{\beta}^{2}\right)\left\{\left(2 \beta^{2}+1\right) \widetilde{\beta}^{2}-3\right\}+x\left\{\left(3 \beta^{2}+1\right) \widetilde{\beta}^{4}-8\left(\beta^{2}+1\right) \widetilde{\beta}^{2}+12\right\}\right] \frac{P^{2}}{Q^{2}} \\
& \left.\left.+\left(3 \beta^{2} x-5 \beta^{2}+x-2\right) \widetilde{\beta}^{4}+2\left\{\left(2 \beta^{2}+1\right) x^{2}-4\left(\beta^{2}+1\right) x+3 \beta^{2}+5\right\} \widetilde{\beta}^{2}-3\left(2 x^{2}-4 x+3\right)\right\}\right], \\
& \left.W_{T S}^{\tau a}\right|_{\mathrm{PM}}=\frac{\alpha}{2 \pi} \delta_{\gamma} \frac{\left(1-\widetilde{\beta}^{2}\right)^{3 / 2}}{\widetilde{\beta}^{3}}\left[L-\frac{2 \beta \widetilde{\beta}}{1-\beta^{2} \widetilde{\beta}^{2}}\right]\left\{1-x-x \frac{P^{2}}{Q^{2}}\right\} .
\end{aligned}
$$

\section{APPENDIX C: THE $\boldsymbol{n}$ th MOMENT OF $\boldsymbol{F}_{1}^{\gamma}$ IN PQCD}

The PQCD prediction for the $n$th moment of $F_{1}^{\gamma}$ up to NLO is summarized as follows:

$$
\begin{aligned}
\int_{0}^{1} d x x^{n-1} F_{1}^{\gamma}\left(x, Q^{2}, P^{2}\right)=\int_{0}^{1} d x x^{n-2}\left(F_{2}^{\gamma}-F_{L}^{\gamma}\right)= & \frac{\alpha}{4 \pi} \frac{1}{2 \beta_{0}}\left[\sum_{i=+,-, N S} \widetilde{P}_{i}^{n} \frac{1}{1+\lambda_{i}^{n} / 2 \beta_{0}} \frac{4 \pi}{\alpha_{s}\left(Q^{2}\right)}\left\{1-\left(\frac{\alpha_{s}\left(Q^{2}\right)}{\alpha_{s}\left(P^{2}\right)}\right)^{\lambda_{i}^{n} / 2 \beta_{0}+1}\right\}\right. \\
& +\sum_{i=+,-, N S} A_{i}^{n}\left\{1-\left(\frac{\alpha_{s}\left(Q^{2}\right)}{\alpha_{s}\left(P^{2}\right)}\right)^{\lambda_{i}^{n} / 2 \beta_{0}}\right\}+\sum_{i=+,-, N S}\left(B_{i}^{n}-\frac{\widetilde{P}_{i}^{(L), n}}{1+\lambda_{i}^{n} / 2 \beta_{0}}\right) \\
& \left.\times\left\{1-\left(\frac{\alpha_{s}\left(Q^{2}\right)}{\alpha_{s}\left(P^{2}\right)}\right)^{\lambda_{i}^{n} / 2 \beta_{0}+1}\right\}+C_{\gamma}^{n}-2 \beta_{0} \delta_{\gamma} B_{\gamma, L}^{n}\right]
\end{aligned}
$$

where $\beta_{0}\left(=11-2 N_{f} / 3\right)$ is the one-loop QCD $\beta$ function and $\alpha_{s}\left(Q^{2}\right)$ is the QCD running coupling constant. All the necessary information on the parameters in the above formula can be obtained from Ref. [21]. The eigenvalues of the oneloop anomalous dimensions, $\lambda_{i}^{n}(i=+,-, N S)$, are given in Appendix A. The parameters $\widetilde{P}_{i}^{n}, A_{i}^{n}, B_{i}^{n}$, and $C_{\gamma}^{n}$, which are relevant to the structure function $F_{2}^{\gamma}$, are given in Appendix B. Finally the parameters $\widetilde{P}_{i}^{(L), n}$ and $B_{\gamma, L}^{n}$ relevant to the longitudinal structure function $F_{L}^{\gamma}$ are given in Appendix C.

The $n$th moment of $g_{1}^{\gamma}$ up to NLO is expressed in a similar form as above and is given in Eq. (3.16) of Ref. [6]. 
[1] R. Nisius, in Photon 2001, Ascona, Switzerland, 2001, hep-ex/0110078 (to be published in the Proceedings) and references therein.

[2] M. Krawczyk, in Photon 2000: International Conference on the Structure and Interactions of the Photon, Ambleside, England, 2000, edited by A. J. Finch, AIP Conf. Proc. No. 571 (AIP, Melville, NY, 2001), pp. 3-17, and references therein.

[3] D. Barber, in Proceedings of the "Zeuthen Workshop on the Prospects of Spin Physics at HERA," edited by J. Blümlein and W. D. Nowak, DESY 95-200, p. 76.

[4] M. Stratmann and W. Vogelsang, Z. Phys. C 74, 641 (1997).

[5] M. Stratmann and W. Vogelsang, Phys. Lett. B 386, 370 (1996).

[6] K. Sasaki and T. Uematsu, Phys. Rev. D 59, 114011 (1999); Phys. Lett. B 473, 309 (2000); Eur. Phys. J. C 20, 283 (2001).

[7] M. Glück, E. Reya, and C. Sieg, Phys. Lett. B 503, 285 (2001); Eur. Phys. J. C 20, 271 (2001).

[8] H. Baba, K. Sasaki, and T. Uematsu, Phys. Rev. D 65, 114018 (2002).

[9] V. M. Budnev, V. L. Chernyak, and I. F. Ginzburg, Nucl. Phys. B34, 470 (1971).

[10] V. M. Budnev, I. F. Ginzburg, G. V. Meledin, and V. G. Serbo, Phys. Rep. 15, 181 (1974).

[11] R. W. Brown and I. J. Muzinich, Phys. Rev. D 4, 1496 (1971).
[12] C. E. Carlson and W. K. Tung, Phys. Rev. D 4, 2873 (1971).

[13] J. Soffer, in SPIN 2000: 14th International Spin Physics Symposium, Osaka, Japan, 2000, edited by K. Hatanaka, T. Nakano, K. Imai, and H. Ejiri, AIP Conf. Proc. No. 570 (AIP, Melville, NY, 2001), p. 461.

[14] K. Sasaki, J. Soffer, and T. Uematsu, Phys. Lett. B 522, 22 (2001).

[15] C. Bourrely, E. Leader, and J. Soffer, Phys. Rep. 59, 95 (1980).

[16] J. Soffer, Phys. Rev. Lett. 74, 1292 (1995).

[17] J. Soffer and O. V. Teryaev, Phys. Lett. B 419, 400 (1998); 490, 106 (2000).

[18] G. Bonneau, M. Gourdin, and F. Martin, Nucl. Phys. B54, 573 (1973); Z. Kunszt and V. M. Ter-Antonyan, Lett. Nuovo Cimento Soc. Ital. Fis. 4, 940 (1972).

[19] M. Glück, E. Reya, and I. Schienbein, Phys. Rev. D 63, 074008 (2001)

[20] M. L. Mangano and S. J. Parke, Phys. Rep. 200, 241 (1991).

[21] T. Uematsu and T. F. Walsh, Nucl. Phys. B199, 93 (1982).

[22] R. Nisius, Phys. Rep. 332, 165 (2000).

[23] Ch. Berger and W. Wagner, Phys. Rep. 146, 1 (1987).

[24] K. Sasaki, Phys. Rev. D 22, 2143 (1980); Prog. Theor. Phys. Suppl. 77, 197 (1983).

[25] A. V. Manohar, Phys. Lett. B 219, 357 (1989). 